Revista de Investigación Educativa 26

enero-junio, 2018 | ISSN 1870-5308 | Xalapa, Veracruz

Instituto de Investigaciones en Educación | Universidad Veracruzana

\title{
La formación en investigación educativa para profesores de informática. Una experiencia cubana
}

\section{Training in educational research for teachers of informatics. A Cuban experience}

\author{
María Amelia Muñoz Pentón ${ }^{a}$ \\ Keila Irene Díaz Tejera ${ }^{b}$ \\ Emma Regina Fierro Martín
}

Recibido: 26 de abril de 2017

Aceptado: 26 de septiembre de 2017

La formación en investigación para profesores de Informática, en el contexto cubano, se convierte en una necesidad de primer orden, debido a la urgencia de perfeccionar el proceso de enseñanza-aprendizaje de esta materia en las instituciones educativas. En la Universidad Central Marta Abreu de Las Villas, Cuba, se ha desarrollado una experiencia que, a partir de la constitución de grupos de trabajo investigativo colaborativo, organizados por la gestión de un proyecto de investigación, ha propiciado la detección y solución de problemas de investigación del proceso de enseñanza-aprendizaje de la Informática en las instituciones educativas. Con esta experiencia, el futuro profesor realiza una práctica profesional especializada, enriquece su labor y eleva su profesionalidad. La producción científica, obtenida del trabajo de los grupos, contribuye al perfeccionamiento del proceso de enseñanza-aprendizaje de la Informática y revela nuevas teorías que enriquecen la Didáctica de la Informática como disciplina pedagógica.

Palabras clave: Formación de profesores; investigación educativa; proyecto de investigación; institución educativa; producción científica.

\footnotetext{
a Doctora en Ciencias Pedagógicas, Profesora de Informática, Universidad Central Marta Abreu de Las Villas, Cuba.凶 mmpenton@uclv.cu

b Doctora en Ciencias Pedagógicas, Profesora de Informática, Universidad Central Marta Abreu de Las Villas, Cuba.

‘ Doctora en Ciencias Pedagógicas, Profesora de Informática, Universidad Central Marta Abreu de Las Villas, Cuba.
} 


\title{
La formación en investigación educativa para
}

profesores de informática. Una experiencia cubana

María Amelia Muñoz Pentón, Keila Irene Díaz

Tejera, Emma Regina Fierro Martín

Research training for computer science teachers becomes a necessity of the first order, in the cuban context, due to the urgency of perfecting the teaching-learning process of this subject in educational institutions. In the Central University Marta Abreu of Las Villas, Cuba, it has developed an experience, from the formation of groups of collaborative work organized by the management of a research project has led to the detection and troubleshooting research of the process learning of Informatics in educational institutions. With this experience, the future professor carries out a specialized professional practice, enriches his work and elevates his professionalism. The scientific production, obtained from the work of the groups, contributes to the improvement of the learning process of Informatics and reveals new theories that enrich the Didactics of Informatics as a pedagogical discipline.

Keywords: Teacher training; educational research; research project; educational institution; scientific production.

\section{La formación en investigación educativa para profesores de informática. Una experiencia cubana}

\author{
Training in educational research for teachers \\ of informatics. A Cuban experience
}

\section{Introducción}

$\mathrm{E}$ 1 desarrollo de las naciones depende ahora más que nunca de la calidad de la formación a la que se accede en las universidades y del conocimiento que se pueda generar y acumular en ellas (Organización de Estados Iberoamericanos [OEI], 2010). Asumir esta meta implica un reto para las generaciones del presente siglo, ya que la creación y acumulación de conocimientos depende de la cantidad y la calidad de las investigaciones que en los distintos campos de las ciencias se sucedan, por lo que se necesita de una sólida formación de los profesionales para la ejecución de investigaciones científicas. 
El reclamo de diversas comunidades educativas sobre conjugar docente e investigador en una misma persona, a partir de su formación científica investigativa, tiene antecedentes tanto en los países del llamado primer mundo como en los de América Latina. La formación del docente como investigador en el caso de aquéllos parte de tres modelos que se complementan: el docente como investigador autónomo, como integrante de un equipo de investigación y como profesional reflexivo (Enríquez, 2007). El estudio de este autor, desde una perspectiva latinoamericana, registra experiencias aisladas que convergen con las descritas en el primer mundo entre las décadas de 1950 y 1960; otras en la década de 1980 sobre el docente como investigador de temas culturales; iniciativas que giran en torno al docente como investigador participativo y al docente como investigador dialéctico.

En el ensayo “¿Qué demonios pasa en las aulas?” (Candela, Rockwell \& Coll, 2009) se aborda una arista de esta temática cuando se plantea la necesidad de valorar el trabajo que realizan los profesores tanto dentro como fuera del aula, antes de juzgarlos. Esta situación puede considerarse común en el contexto educativo independientemente del país, pues en el caso cubano también se hace referencia a

entornos institucionales que restringen el tiempo, el espacio y los recursos, que imponen un currículo estándar con grados variables de control, sometiéndolos a diversos grados de presión para que se ajusten a unos modelos que pueden no ser de su elección. (p. 6)

Dichos autores plantean que aunque las acciones de los maestros en correspondencia con las perspectivas educativas sean diferentes a las del investigador, incorporar a estos al proceso de análisis también puede ayudar a construir una teoría más cercana a la experiencia del aula.

Analizando cómo se vislumbra hoy en algunos países la formación del docente investigador y cómo se proyectan las instituciones encargadas de la formación de profesores en esta dirección, emerge la necesidad de educar al futuro docente en la práctica de la investigación educativa desde su formación inicial en la universidad. El trabajo, desde esta perspectiva, posibilita "darle una mirada pedagógica a la situación universitaria de nuestro tiempo - a la idea de Universidad-, marcada por su orientación profesionalizante y científico-técnica” (Runge, 2005, p. 3).

La experiencia cubana en este campo aporta ideas interesantes que pueden constituir punto de partida para la organización y planificación de los procesos de investigación educativa en otros contextos. 


\section{La formación de profesores en investigación. Visión desde la educación cubana}

La concepción de un docente investigador tiene sus antecedentes en el proyecto inglés “Humanities Curriculum Project", liderado por Stenhouse (1971) y desarrollado en el Reino Unido entre 1967 y 1968, que buscaba que no fueran más los investigadores sociales, externos al contexto del plantel escolar, los que determinaran las acciones del sistema educativo, y menos aún, los que determinaran el quehacer de los maestros desde una perspectiva lejana de la realidad escolar; sino que fuese el propio maestro quien se articulara con los contenidos de la ciencia y de la cultura, para no ser sólo un instrumento del currículo, sino un gestor y ejecutor de éste, posibilitando así el cambio.

Desde esta revolucionaria perspectiva, se sientan las bases para las disímiles reformas educativas en la formación del docente en muchos de los países del mundo, donde cada día se trata, más que de formar investigadores para la docencia, de formar docentes investigadores capaces de transformar su propia práctica con el empleo del método científico, en procesos de investigación liderados y promovidos por ellos mismos.

Ideas muy similares plantea Martínez (2014), al afirmar que "se reivindica la docencia como profesión con todas las características que las carreras profesionales tienen, tales como una sólida formación en un núcleo de conocimientos comunes, capacidad para tomar decisiones y formación en investigación” (p. 64).

El profesor no puede tener como propósito contemplar la realidad educativa, sino conocerla mediante la propia práctica, lo que le permite realizar valoraciones adecuadas para después transformarla. Al ejecutar junto a la docencia la búsqueda científica y la solución de problemas del proceso de enseñanza aprendizaje, el docente realiza una práctica social especializada, enriquece su labor y eleva su profesionalidad (Addine, González \& Recarey, 2003).

En Cuba, a partir de 2006, se introduce en el currículo de todas las carreras encargadas de la formación de profesores una disciplina cuyo contenido fundamental es la preparación de los futuros docentes en investigación educativa. El contenido del proceso de enseñanza-aprendizaje de la investigación educativa incluye "los conocimientos científicos pedagógicos, que permiten el análisis sistemático de la práctica educativa desde posiciones científicas, las habilidades científico-investigativas que posibilitan operar con la ciencia en el contexto educativo y los valores ético profesionales" (Chirino, 2002, p. 19). 
El conocimiento científico pedagógico es condición para el desarrollo de habilidades científico investigativas. Asumiendo el criterio de Carballo (2002), las habilidades investigativas son habilidades generales que deben formar parte del perfil profesional de cada carrera, que le permiten al estudiante enfrentar los problemas de la práctica profesional y darle solución con iniciativas.

Significa que en cada una de las carreras se concibe la formación investigativa del profesional sobre bases teóricamente fundamentadas, revelando en su concepción, la lógica interna de aquellas interrelaciones entre los componentes del proceso que puedan distinguirla y caracterizarla, en las que subyacen las aspiraciones sociales para con el docente que se forma, requiriéndose, para conseguir este objetivo, investigar desde dentro el comportamiento ideal de estos componentes y la estructuración del sistema de relaciones que se establece entre ellos, partiendo del modelo del profesional.

\section{La formación investigativa de profesores de Informática}

El problema profesional del futuro profesor de Informática en el contexto cubano se concreta en la Educación informática de las nuevas generaciones atendiendo a las características particulares de los escolares de cada nivel educativo, por lo que posee un amplio espectro en su desempeño profesional.

La formación investigativa de este profesional se convierte en una necesidad de primer orden debido a la urgencia de promover y desarrollar investigaciones educativas relativas al proceso de enseñanza-aprendizaje de la Informática, proceso que aún necesita de la consolidación de teorías científicamente fundamentadas, dada su naturaleza versátil influida por los vertiginosos cambios que se producen en la tecnología, que requieren del perfeccionamiento sistemático de su didáctica particular. "Los problemas objeto de investigación referentes al desarrollo de procedimientos y métodos más efectivos para la enseñanza de la Informática tienen gran prioridad, importancia y actualidad” (Oceguera, Expósito, Díaz \& Bonne, 2009, p. 18).

Tomando como referente la concepción didáctica para la formación investigativa del profesor de Informática propuesta por Ross (2014), se plantea como exigencia imprescindible de este proceso la identificación y solución de problemas de investigación, estableciendo la unidad entre los problemas que en teoría demanda el proceso de enseñanza-aprendizaje de la Informática y los problemas prácticos existentes en las instituciones educativas. 
La identificación y solución de problemas asociados al proceso de enseñanzaaprendizaje de la Informática requieren orientación hacia necesidades de investigación que se convierten en líneas, las que deben corresponderse con aquellas invariantes que, en términos de proceso investigativo, se presentan al futuro profesor de Informática en su práctica profesional y devienen en objetos de investigación:

¿Cuáles pueden ser esas líneas de investigación?

¿Cómo detectar en los planteles escolares las necesidades de investigación asociadas a estas líneas?

¿Cómo orientar la producción científica de la universidad hacia la solución de los problemas asociados a esas necesidades?

\subsection{La organización de la formación investigativa de futuros profesores de Informática en una universidad cubana}

En la Universidad Central Marta Abreu de Las Villas, en el departamento encargado de la formación de profesores de Informática, se gestiona desde 2011 un sistema de proyectos de investigación que funcionan a partir de la constitución de grupos de trabajo investigativo colaborativo, en los cuales se organizan los estudiantes para el desarrollo de procesos de investigación. Con estas investigaciones se promueve la identificación y solución de problemas en función de la situación particular de las instituciones educativas en que estos estudiantes realizan su práctica profesional.

Estos grupos se constituyen en el tercer año de la formación inicial, funcionan sobre la base del aprendizaje colaborativo y la reflexión colectiva, favoreciendo la identificación y solución de problemas de investigación asociados al proceso de enseñanza-aprendizaje de la Informática, y la producción científica se orienta, de manera creativa, al perfeccionamiento de este proceso en las instituciones educativas.

Las experiencias del trabajo de los grupos, bajo la dirección de los docentes universitarios encargados de su formación, permiten hacer una caracterización del estado actual del proceso de enseñanza-aprendizaje de la Informática en cada uno de los niveles educativos en que se insertan los estudiantes para la realización de su práctica profesional.

La caracterización, que se actualiza cada año, aporta los principales problemas a resolver en las instituciones educativas, que conjugados con las demandas de la Didáctica de la Informática, permiten establecer invariantes, consideradas de esta manera debido a que pueden constituir objetos de investigación, independientemente del contenido que se trate en cada nivel educativo y de las características de los educandos. 
Estas invariantes, formuladas como líneas de investigación a priorizar en el área de la educación en Informática, se centran en:

- El tratamiento de las formas regulares de la enseñanza de la Informática (formación de conceptos informáticos, elaboración de procedimientos algorítmicos y resolución de problemas).

- La estructuración de la clase de Informática bajo los diferentes enfoques didácticos de la enseñanza.

- El diseño de aplicaciones educativas.

- El diseño de estrategias de aprendizaje con recursos informáticos.

El tratamiento de las formas regulares y la estructuración de la clase de Informática bajo los diferentes enfoques didácticos de su enseñanza, son líneas de investigación que conducen, por regla general, a la obtención de una producción científica compuesta por propuestas didácticas y metodológicas para el perfeccionamiento del proceso de enseñanza de esta asignatura, tales como: sistemas de ejercicios o de problemas, sugerencias metodológicas, sistemas de tareas docentes, entre otras.

El diseño de aplicaciones educativas, por su parte, aporta propuestas de productos tecnológicos en diferentes formatos, los cuales, como medio de enseñanza, persiguen objetivos educativos particulares adecuados al problema que resuelven. Se obtienen así: software educativos, productos multimedia, páginas y sitios web, que pueden generarse con diversas herramientas de autor o software profesionales específicos.

El diseño de estrategias de aprendizaje con recursos informáticos, aporta propuestas didácticas que permiten la orientación, ejecución y control del proceso de enseñanza y aprendizaje mediado por la computadora como vehículo de enseñanza fundamental. Se obtienen a partir de esta línea de investigación: sistemas de tareas para el empleo, ya sea de software educativo o de alguna aplicación educativa que haya sido diseñada con un objetivo particular. Pueden obtenerse, además, propuestas de webquest, cazatesoros y unidades didácticas que promuevan un aprendizaje productivo en los educandos.

Dichas líneas constituyen el eje central del desarrollo de la investigación educativa en el área de la Informática desde la universidad hacia las instituciones educativas, de manera que una vez graduado, el profesor, como parte de su formación permanente, está preparado para caracterizar el estado del proceso de enseñanza-aprendizaje en que se desempeña, detectando nuevos problemas de investigación y proponiendo las soluciones adecuadas. 


\section{Experiencias de la solución de problemas del proceso docente en las instituciones educativas a partir de la formación investigativa de futuros profesores}

Se describe la experiencia del trabajo desarrollado por un grupo de siete profesores y 34 estudiantes divididos en cinco grupos de trabajo investigativo colaborativo.

Se realizó una caracterización del proceso de enseñanza-aprendizaje de la Informática en varias instituciones educativas de la ciudad de Santa Clara, correspondientes a diferentes niveles de educación (primario, secundario, preuniversitario y técnico profesional). Dicha caracterización responde a un grupo de dimensiones para la evaluación de las cuales se determinaron las correspondientes categorías:

\section{Dimensión: Dirección del aprendizaje}

- Evidencia de la utilización de los enfoques didácticos para la enseñanza de la informática.

- Selección de métodos adecuados al nivel educativo.

- Presencia de estrategias para la formación y fijación de conceptos y procedimientos.

- Empleo del software educativo como medio de enseñanza.

- Utilización de formas de organización de la clase adecuadas al nivel educativo.

- Utilización de variedad de formas de evaluación.

- Utilización del software en la evaluación del aprendizaje.

\section{Dimensión: Aprendizaje}

- Asimilación de conceptos informáticos.

- Asimilación de procedimientos informáticos.

- Desarrollo de habilidades para la resolución de problemas con el empleo de la computadora.

\section{Dimensión: Condiciones objetivas}

- Condiciones ambientales de los laboratorios (mobiliario, iluminación, ventilación). 
- Estado de instalación de los software educativos.

- Horarios establecidos para clases de la asignatura por grados.

\section{Dimensión: Trabajo científico metodológico del colectivo de la institución educativa}

- Existencia de estrategias de aprendizaje de la asignatura en función del diagnóstico.

- Identificación de problemas en el proceso de enseñanza aprendizaje de la Informática, por parte de los agentes directivos de las instituciones educativas.

- Existencia de temas de investigación en la institución que respondan al proceso de enseñanza aprendizaje de la Informática.

Se utilizaron como métodos de investigación: el análisis documental, la observación, la encuesta, la entrevista y la prueba pedagógica. Los instrumentos elaborados para la recolección de la información empírica fueron conformados y sometidos a pilotaje, teniendo en cuenta las características de la asignatura y las particularidades de estudiantes y profesores de cada nivel educativo. Se identificaron los siguientes problemas:

- Los agentes directivos no identifican problemas asociados al proceso de enseñanza-aprendizaje de la Informática que sí fueron detectados durante el proceso de exploración y diagnóstico de la investigación.

- No se aprecia una concepción didáctica coherente de la clase de Informática, atendiendo a las diferencias individuales y el adecuado empleo del software educativo como medio de enseñanza.

- Los estudiantes demuestran bajos niveles de aprendizaje, que se focalizan en la asimilación de conceptos y procedimientos básicos, así como en la integración y aplicación de los conocimientos adquiridos a la solución de problemas prácticos.

- No existe en las instituciones educativas una estrategia organizada que garantice el empleo de la computadora como medio de enseñanza y como herramienta de trabajo.

A partir de la identificación de estos problemas, el trabajo de los grupos se orientó a la planificación del proceso de investigación sobre la base de problemas científicos más concretos, contextualizados en instituciones educativas específicas. De las investigaciones se deriva la producción científica individual de cada estudiante, cuyos 
resultados responden a necesidades de los diferentes niveles educativos y se pueden resumir en los siguientes:

- Objetos de aprendizaje para la formación de conceptos en la educación primaria.

- Ejercicios orientados a la fijación de conceptos y elaboración de procedimientos informáticos en las educaciones secundaria y preuniversitaria.

- Propuesta de ejercicios y problemas para el desarrollo de habilidades informáticas.

- Software para la enseñanza aprendizaje del Sistema Operativo GNU/Linux en la educación preuniversitaria.

- Webquest, unidades didácticas y objetos de aprendizaje para la resolución de problemas informáticos en la educación preuniversitaria.

Todos los resultados elaborados fueron validados en las instituciones educativas correspondientes, contribuyendo al perfeccionamiento del proceso de enseñanzaaprendizaje, facilitando la planificación de las actividades docentes por parte de los docentes y favoreciendo el aprendizaje de los estudiantes. Se presentan a continuación dos de estos resultados:

- Objetos de aprendizaje para la formación de conceptos y elaboración de procedimientos informáticos por escolares de cuarto grado.

Esta investigación parte de las limitaciones de escolares de cuarto grado en la asimilación de conceptos y procedimientos informáticos. Tomando como objeto de investigación el proceso de enseñanza-aprendizaje de la Computación, se proponen como objetos de aprendizaje presentaciones electrónicas y ejercicios interactivos. Las primeras, se emplean para comunicar el contenido al escolar a partir de relatos narrados por "historietas" (Figura 1).

En una historia, contada por personajes asociados al tema de la clase, se abordan de una manera atractiva los conceptos y procedimientos informáticos a asimilar para la posterior resolución de problemas. De cada una de las historietas se derivan ejercicios interactivos, a partir de los cuales los escolares pueden autoevaluarse y fijar los conceptos y procedimientos correspondientes. 


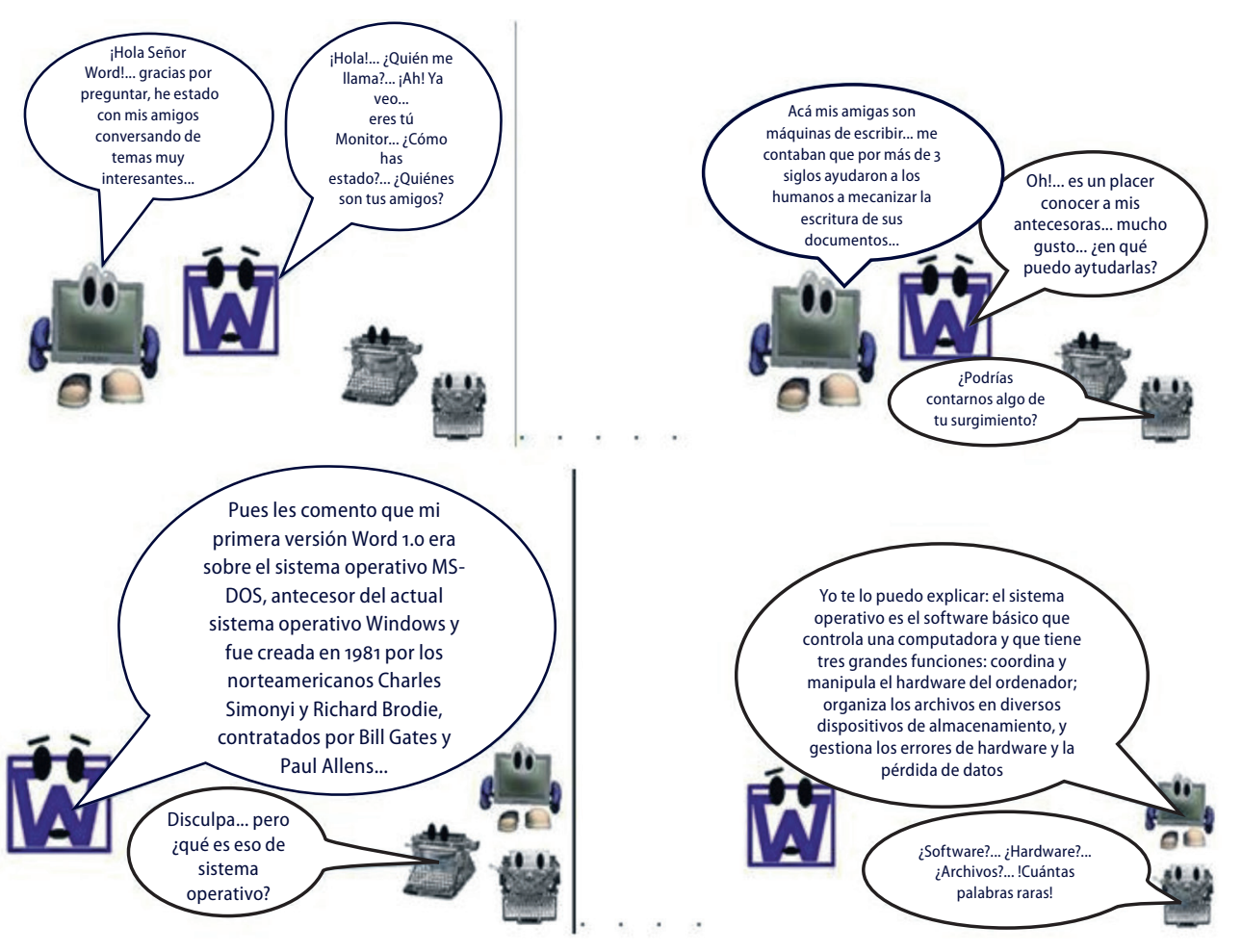

Figura 1. Ejemplo de una de las páginas de la historieta

Fuente: Rodríguez, 2014.

- Mapas conceptuales para la formación de conceptos informáticos.

Esta investigación partió de las insuficiencias detectadas en la asimilación de conceptos informáticos en escolares de sexto grado. Teniendo como objeto de investigación el proceso de enseñanza-aprendizaje de la Computación, se plantea como propuesta de solución el empleo de mapas conceptuales para contribuir a este proceso.

Los mapas conceptuales elaborados con el empleo de la herramienta Cmap Tools (Figura 2) se conciben como recursos, métodos y estrategias en el aprendizaje del escolar. Aprovechando las potencialidades de la propia herramienta, a cada mapa le han sido incorporados recursos en diferentes formatos que facilitan la estructuración del mapa mental del escolar, a partir de un sistema de conceptos asociados a un contenido informático específico. 


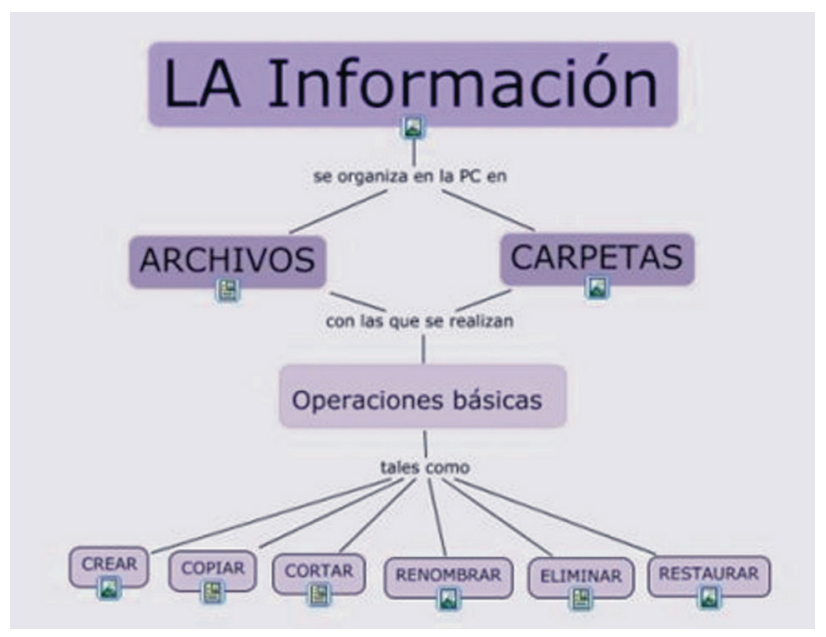

Figura 2. Ejemplo de una de los mapas conceptuales

Fuente: Esquirol, 2015.

\section{Conclusiones de la experiencia}

Al ejecutar, junto a la docencia, la búsqueda científica y la solución de problemas de investigación asociados al proceso de enseñanza-aprendizaje de la Informática, el futuro profesor realiza una práctica profesional especializada, enriquece su labor y eleva su profesionalidad.

La Didáctica de la Informática como disciplina pedagógica necesita de una actualización permanente, dada la contradicción no resuelta entre la rapidez de los cambios que se producen en el desarrollo de las tecnologías de la información y la comunicación y el desarrollo de metodologías para su incorporación al proceso docente educativo.

De la organización del proceso de formación investigativa en la universidad y de las demandas de las didácticas particulares, depende el desarrollo de las investigaciones en las instituciones educativas, que en el área de la Informática se pueden concretar en el tratamiento de las formas regulares de la enseñanza de la Informática, la estructuración de la clase de esta asignatura a partir del empleo adecuado de los enfoques didácticos que se sugieren para su enseñanza, el diseño de aplicaciones educativas y de estrategias de aprendizaje con recursos informáticos. 
La producción científica que se deriva de estos procesos de investigación contribuye al perfeccionamiento del proceso de enseñanza-aprendizaje de la Informática y revela nuevas teorías que enriquecen la Didáctica de la Informática como disciplina pedagógica.

Esta estrategia de trabajo colaborativo facilitó el intercambio entre tutores y estudiantes y entre los propios estudiantes, favoreciendo la apropiación y construcción colectiva del conocimiento, contribuyendo a la motivación y la estimulación de la capacidad para formular preguntas, enunciar problemas y buscar soluciones efectivas.

\section{Lista de referencias}

Addine, F., González, A., \& Recarey, S. (2003). Principios para la dirección del proceso pedagógico. En Problemas de la enseñanza y el aprendizaje. Compendió de Pedagogía (pp. 80-101). La Habana: Pueblo y Educación.

Candela, A., Rockwell, E., \& Coll, C. (enero-junio, 2009). ¿Qué demonios pasa en las aulas? La investigación cualitativa del aula. CPU-e, Revista de Investigación Educativa, 8, 1-28. Recuperado de http://revistas.uv.mx/index.php/cpue/article/view/81

Carballo, M. (2002). Estrategia pedagógica para el desarrollo de habilidades investigativas en estudiantes de la Licenciatura en Educación especialidad Agronomía (Tesis doctoral inédita). Universidad de Ciencias Pedagógicas Félix Varela, Santa Clara, Cuba.

Chirino, M. (2002). El perfeccionamiento de la formación inicial investigativa de los profesionales de la educación (Tesis doctoral inédita). Universidad de Ciencias Pedagógicas Enrique José Varona, La Habana.

Esquirol, M. (2015). Mapas conceptuales para el aprendizaje de conceptos informáticos por escolares de sexto grado (Tesis de licenciatura inédita). Universidad de Ciencias Pedagógicas Félix Varela, Santa Clara, Cuba.

Enríquez, P. (2007). El docente-investigador: Un mapa para explorar un territorio complejo. Argentina: Lae.

Martínez, M. C. (enero-junio, 2014). Reflexiones en torno a la Investigación-Acción educativa. CPU-e, Revista de Investigación Educativa, 18, 58-86. Recuperado de http://revistas.uv.mx/index.php/cpue/article/view/756

Oceguera, S., Expósito, C., Díaz, G., \& Bonne, E. (2009). Metodología de la enseñanza de la Informática. La Habana: Educación Cubana. 
Organización de Estados Iberoamericanos. (2010). Metas educativas 2021. La educación que queremos para la generación de los bicentenarios. España: Autor.

Rodríguez, S. (2014).Objetos de aprendizaje para la formación de conceptos y elaboración de procedimientos informáticos por escolares del 4to. Grado (Tesis de licenciatura inédita). Universidad de Ciencias Pedagógicas Félix Varela, Santa Clara, Cuba.

Ross, I. (2014). La formación investigativa del profesional de la educación especialidad Informática en universidades de ciencias pedagógicas (Tesis doctoral inédita). Universidad de Ciencias Pedagógicas Félix Varela, Santa Clara, Cuba.

Runge, A. (2005). Reflexiones pedagógicas sobre la investigación y la docencia universitarias a la luz de la formación: el modelo humboldtiano como ejemplo. Uni-Pluri/versidad, 5(2), 41-49. Recuperado de http://aprendeenlinea.udea. edu.co/revistas/index.php/unip/article/view/12151

Stenhouse, L. (1971). The Humanities Curriculum Project: The Rationale. Theory into Practice, 10(3). doi:http://dx.doi.org/10.1080/00405847109542322 